wheezing, bronchitis and laryngitis. 203 nasopharyngeal swabs were collected during epidemic season 2019-2020, 180 $(88,7 \%)$ of them were positive: RV - 56 (31,7\%), AdV - 39 (22,2\%), CoVNL-63, 229E - 28 (12,7\%), hBoV - 19 $(10,6 \%)$, IVA -15 (8,3\%), PIV - 12 (6,6\%). Confirmed diagnosis of pneumonia had $6(10,6 \%)$ children with RV. 10 $(17,8 \%)$ children with AdV infection had pneumonia as a clinical manifestation. CoVNL-63, 229E caused rhinopharyngitis, croup, wheezing, tracheobronchitis. Generally, 103 samples were collected during the period from March 2019 until September 2019. 98 of them were positive: RSV -37 (37,8\%), RV -29 (29,6\%), CoV - 12 (12,2\%), AdV - 10 (10,2\%), PIV - $9(9,2 \%)$.

Conclusions There has been a change in the etiological spectrum of ARVI during the last two years. During epidemic season 2018-2019 in Ukraine, the most prevalent viruses were hMPV -33 (26,3\%), IVA -28 (22,4\%), RV - 21 (16,8\%). During epidemic season 2019-2020 the most commonlydetected pathogens were RV- 56 (31,7\%), AdV 39 (22,2\%), CoVNL 63, 229E28 (12,7\%).

Epidemiological studies should be continued in order to forecast the probable clinical course of disease, and for optimization therapy.

\section{THE ADOLESCENT SLEEP CRISIS: THE CAUSES, CONSEQUENCES AND SOLUTIONS}

Ffion Byrne*. University of Bristol

10.1136/archdischild-2021-europaediatrics.59

This paper analyzes how reduced sleep duration and quality, referred to as sleep deprivation, affects adolescent development, with a particular interest in associations with obesity and mental health.

To answer this question, a literature search was conducted that produced 12 suitable papers for analysis. These papers were read and reviewed to identify the main findings which were then collated to find common themes.

The results showed that there were five main developmental areas affected by sleep deprivation: academic achievement, obesity, social jetlag, mental health and parent-adolescent relationship.

This study emphasizes the need for greater research into the effects of adolescent sleep deprivation in UK samples. It also highlights three ways in which adolescent sleep deprivation could be prevented: earlier school start times, sleep education and parental education.

\section{IMPACT OF COVID - 19 ON ONCOLOGY PATIENTS IN IRELAND}

Livija Ebner*, Sana Marium Ali, Muhmmad Azam. Wexford General Hospital, HSE

10.1136/archdischild-2021-europaediatrics.60

Background Cancer is a major cause of mortality in Ireland. The total number of cases will almost double by 2040. 1 in 3 people of all age group will develop cancer in their lifetime. On average 137 cases of cancer were diagnosed per year among children under the age 15 during the 21 year period.
211 cases of cancer were diagnosed per year under the age of 20. Five year survival has average $81 \%$ in most recent 10 years. Majority of survivors had been diagnosed with Leukemia $31 \%$. Brain and CNS Tumour's surviving rate is $23 \%$.

Aims To analyse the impact of COVID -19 on Oncology patients. Reason of Hospital admission during COVID-19 Pandemic in 2020-2021 in Wexford General Hospital. To aim for excellent patient care of Oncology patients and to improve access, quality and value for all children with Oncology issue in Wexford area. Assess the resources given to care for these inpatients and changes of workload in last year in COVID 19 Pandemic.

Standards Assessment of COVID-19 impact on Oncology patients if there is any relation to their hospital admission and sick days, has there been any relation of newly diagnosed Oncology patients due to COVID-19.

Difficulties of high risk Oncology patients in Paediatric Ward during COVID-19 Pandemic. The need of properly trained staff to deal with the workload during COVID-19 Pandemic with proper safety and healthcare to the staff and patient. Implementation of new National Cancer Strategy CHI Crumlin following the standards of treatment of Oncology patient according to $\mathrm{CHI}$ guidelines. National Model of care in Paediatric Oncology Patient Services in Ireland.Concept of integrated Multidisciplinary Care.

Methodology A HIPE search was completed for oncology patients admitted in Wexford General Hospital from January 2020 - January 2021 inclusive. All charts were reviewed and appropriateness of inclusion in the Study was confirmed. Following data was collected. Chart review. Date of birth. Age of diagnosis. Date of admission, cause of admission and diagnosis. Length of stay in hospital. Inclusion of Covid 19 PCR test and its outcome of Oncology patients. Newly diagnosed cases. To rule out if there is any correlation of Covid 19 in newly diagnosed and previously diagnosed patients causing them to be admitted in the hospital in Wexford area. Gender and age breakdown of oncology patients. Transfusion rate, most common cause of infection causing increase in number of bed days and hospital admission.

Result Total number of Oncology patient in Wexford area is 20. 9 oncology patients were admitted from January 2020 to January 2021 out of which 6 new and 3 previously diagnosed oncology patient. Total 12 male oncology patients and 8 female patients in total. 6 newly diagnosed oncology patient from( January 2020 to January 2021), out of which 3 ALL( Acute Lymphoblastic Leukemia),1 Pilocytic Astrocytoma,1 Medulloblastoma,1 Wilms tumor respectively. Peripheral blood culture was tested negative for all inpatients except 1 that was likely contaminant sample. Blood transfusion was given only to ALL patients, $46 \%$ pack RBC was transfused and 54\% platelet transfusion was given to patient admitted in Wexford General Hospital in Paeds ward. 6 patients were transferred to Tertiary care hospital as they were newly diagnosed oncology patients and 3 were not transferred as they had recurrent infection treated in local hospital. Total 9 admissions from January 2020 till January 2021, all patients were tested for COVID 19 PCR and all were negative. Total number of bed days occupied are 93 days out of which ALL patients occupied most days i.e 60 days, Medulloblastoma 31 days, Astrocytoma 2 days respectively. 11 times oncology patients admitted with line infection with febrile neutropenia whereas 